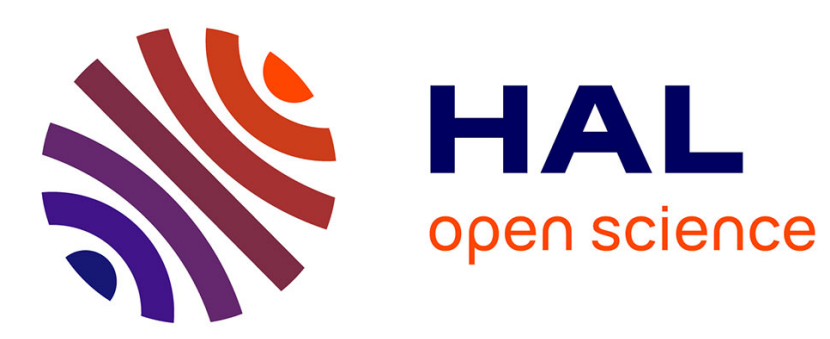

\title{
Quelles valeurs se cachent derrière la motivation des jeunes étudiants-entrepreneurs?
}

\author{
Ines Gabarret, Benjamin Vedel, Pascal Etzol
}

\section{To cite this version:}

Ines Gabarret, Benjamin Vedel, Pascal Etzol. Quelles valeurs se cachent derrière la motivation des jeunes étudiants-entrepreneurs?. Gestion 2000, 2016. hal-01891948

\section{HAL Id: hal-01891948 \\ https://hal.science/hal-01891948}

Submitted on 10 Oct 2018

HAL is a multi-disciplinary open access archive for the deposit and dissemination of scientific research documents, whether they are published or not. The documents may come from teaching and research institutions in France or abroad, or from public or private research centers.
L'archive ouverte pluridisciplinaire HAL, est destinée au dépôt et à la diffusion de documents scientifiques de niveau recherche, publiés ou non, émanant des établissements d'enseignement et de recherche français ou étrangers, des laboratoires publics ou privés. 


\section{Quelles valeurs se cachent derrière la motivation des jeunes étudiants- entrepreneurs ?}

Inès GABARRET (personne de contact)

Enseignant-chercheur, HDR

OCRE, Labex Entreprendre

EDC Paris Business School, Paris, France

ines.gabarret@edcparis.edu

Benjamin VEDEL

Maître de Conférences

LEM-CNRS (UMR 9221), Labex Entreprendre

IAE Lille, Lille, France

Pascal ETZOL

Docteur en Education

The George Washington University, Washington DC, USA

\section{Résumé}

Cet article, à visée exploratoire, s'intéresse à la motivation entrepreneuriale d'un groupe d'étudiantsentrepreneurs de la génération Y en France, et tente de déchiffrer les valeurs véhiculées au travers de leur discours motivationnel. Traditionnellement, la motivation pour créer une entreprise est étudiée par l'approche exclusive push/pull et est reliée à des facteurs individuels. Or, des recherches récentes 
considèrent la motivation comme une combinaison de facteurs et notent des influences environnementales sur la décision de devenir entrepreneur. Nos résultats montrent que la motivation des jeunes étudiants entrepreneurs est complexe et renferme des valeurs associées à leur vision du travail, de la stabilité et de l'autonomie.

\title{
Mots clés :
}

Génération Y; Motivation entrepreneuriale; Valeurs ; Entrepreneuriat; Millennials, Jeunes entrepreneurs, Push-pull, étudiants-entrepreneurs

\begin{abstract}
What values behind the motivation of young students-entrepreneurs?

This exploratory article aims to understand the entrepreneurial motivation of a group of studentsentrepreneurs of the $\mathrm{Y}$ generation in France, and tries to discover the values conveyed through their motivational speeches. Traditionally, the motivation to start a business is studied by the exclusive push and pull approach and is connected to individual factors. However, recent articles consider motivation as a combination of factors and note environmental influences on the decision to become an entrepreneur. Our results show that motivation of young entrepreneurs is complex and contains values associated with their vision of work, stability and autonomy.
\end{abstract}

\section{Keywords :}

Y Generation; Entrepreneurial Motivation ; Entrepreneurship ; Millennials, Young entrepreneurs, Push-pull, Students-entrepreneurs 


\section{Introduction}

Depuis une vingtaine d'années, l'entrepreneuriat ne cesse de se développer et d'évoluer en France. Ces changements s'observent de multiples manières : les cours d'entrepreneuriat se sont largement démocratisés et l'aide aux entreprises en création via les structures d'accompagnement est devenue la norme. De nouveaux statuts apparaissent régulièrement et participent un peu plus à la diffusion de la culture entrepreneuriale (eg. auto-entrepreneur). Le programme d'étudiant-entrepreneur (PEPITE ${ }^{1}$ ) complète l'effort entreprit. Grâce à ce dernier, les établissements de l'enseignement supérieur donnent dorénavant la possibilité aux jeunes de créer leurs entreprises directement pendant leurs années d'étude. Ces initiatives restent importantes, la jeunesse en France faisant face à un environnement économique difficile (chômage élevé ${ }^{2}$ ). Dans le même temps, la création d'entreprise n'a jamais été aussi attirante pour cette population. D'après l'Institut National de la Jeunesse et de l'Education Populaire (INJEP), en 10 ans, le nombre d'entreprises créées par les jeunes a quasiment triplé (125 000 entreprises en 2014). Cette multiplication d'entreprises pose la question des caractéristiques de ces jeunes créateurs et nous amène au sujet de la génération Y.

Concernant la génération Y (aussi appelée millénaires, génération du net, e-génération, etc.), des articles abondent dans les médias, contrairement aux recherches scientifiques, qui sont moins prolifiques. Ce n'est que tout dernièrement que des études sur ce sujet ont commencé à apparaître, certaines d'entre elles allant même jusqu'à questionner l'existence de ce phénomène générationnel (Pichault, Pleyers, 2010). Définir une génération est une tâche difficile. En général, les démographes considèrent les générations par rapport à l'année de naissance. Les historiens, pour leur part, le font en relation à une histoire commune vécue par un groupe (Brillet et al., 2012 ; Bayad et al., 2013). Une génération couvre habituellement des périodes d'une vingtaine d'années (Pichault, Pleyers, 2010). En ce qui concerne la délimitation temporelle de la dite «génération Y », il existe peu de consensus sur le sujet (Minifie, Otto, 2011 ; Pichault, Pleyers, 2010).

\footnotetext{
${ }^{1}$ Pôles Etudiants pour l'Innovation, le Transfert et l'Entrepreneuriat.

${ }^{2}$ D'après Eurostat (www.ec.europa.eu), le taux de chômage des moins de 25 ans atteint 26,1\% en 2013.
} 
La génération Y est considérée dans les médias comme une génération « entrepreneuriale ». Cependant, d'après plusieurs auteurs (Claire, 2012 ; Holt et al., 2012), il s'agirait d'un entrepreneuriat différent de celui étudié dans la littérature classique. Ainsi, les jeunes entrepreneurs d'aujourd'hui ne cherchent pas nécessairement le seul succès économique. Ils sont, en même temps, attirés par d'autres valeurs, telles qu'avoir une vie équilibrée, un impact au sein de leur communauté d'appartenance ou de poursuivre une passion (Claire, 2012).

Dans cet article, nous nous intéressons aux facteurs motivationnels des jeunes étudiantsentrepreneurs de la génération Y et aux valeurs qui y sont rattachées. D’un point de vue théorique, la motivation entrepreneuriale a été expliquée principalement grâce au modèle «Push/Pull»: les individus sont soit attirés (au travers la découverte d'une opportunité d'affaires), soit poussés vers la création d'entreprise (afin de créer leurs propres emplois) (Schjoedt, Shaver, 2007; Gilad, Levine, 1986). Cette approche a été régulièrement remise en cause par un nombre croissant d'auteurs qui considèrent la motivation entrepreneuriale comme un construit complexe (Kirkwood, Cambell-Hunt, 2007).

Concernant les valeurs, les recherches ont d'abord étudié les influences du contexte et de la culture sur l'intention de devenir entrepreneur (Schlaegel et al., 2013). La culture est constituée de valeurs partagées par un groupe social. Elle peut être influencée par les environnements économique et politique. Par exemple, Liñan (2013) montre l'existence d'une relation entre les actions gouvernementales pour développer la création d'entreprise et l'apparition des nouvelles valeurs dans la population.

Pour comprendre la complexité de la motivation entrepreneuriale et des valeurs partagées dans le cas des étudiants-entrepreneurs de la génération Y, nous avons mis en place une étude exploratoire basée sur des entretiens semi-directifs (Miles, Huberman, 1994) au sein d'une grande école de commerce française. Tous les étudiants en dernière année d'études, ayant créé leur entreprise, ont été interrogés. 
Les résultats de la recherche confirment que la motivation est un construit complexe composée de facteurs aussi bien «internes/externes », que «push/pull», et soulignent que ces facteurs reflètent une conception particulière des valeurs comme le travail, la stabilité et l'autonomie des jeunes entrepreneurs Y.

Notre article est organisé de la manière suivante: une première partie développe l'approche «push/pull » de la motivation entrepreneuriale et les caractéristiques de la génération Y dans la littérature. La deuxième partie explique la méthodologie qualitative utilisée et présente le terrain étudié. Dans la troisième partie nous déployons et discutons nos résultats.

\section{La motivation entrepreneuriale}

La motivation entrepreneuriale est souvent étudiée au travers de l'approche «push/pull », laquelle a donné naissance aux concepts d'entrepreneur par opportunité (pull) et par nécessité (push). Tandis que la dimension «push»s'intéresse à la nécessité et à l'insatisfaction au travail (Brockhaus, 1980; Bradley, Roberts, 2005), la dimension «pull» est reliée à la recherche d'opportunités d'affaires (Reynolds et al., 2002; Acs et al., 2005) et au désir d'indépendance (Hughes, 2003; Hessels et al., 2008). Dans ces approches, le choix des individus est mutuellement exclusif, les individus sont soit push, soit pull. Pour dépasser cette limite, plusieurs auteurs indiquent que la motivation entrepreneuriale est un construit complexe (Kirkwood, Cambell-Hunt, 2007) et pour cela elle doit être étudiée comme un continuum entre ces deux extrêmes (Hughes, 2003; Verheul et al., 2010).

Un autre aspect de la littérature s'intéressant à la motivation entrepreneuriale concerne la distinction entre les variables économiques et non économiques. Initialement, une partie importante de la littérature en entrepreneuriat assume que la décision de commencer une affaire est la résultante d'un processus de maximisation dans lequel l'individu compare les revenus des différentes activités et choisi celle qui lui procurera la meilleure rentabilité (Arenius, Minniti, 2005). Néanmoins, les recherches ont aussi montré que des motivations non-économiques, telles que le besoin 
d'accomplissement, la capacité à créer son propre emploi, et le désir d'autonomie, sont souvent rangés premiers dans les facteurs motivationnels (Verstraete, Saporta, 2006).

Dans l'approche push/pull, les facteurs motivationnelles restent classiquement au niveau individuel. Williams et Williams (2012) dépassent cette vision et indiquent que la motivation entrepreneuriale peut être influencée par le contexte socio-spatial. Liñan et al. (2013) étudient les relations entre la croissance économique, la motivation entrepreneuriale et la culture. Selon eux, il existe une relation entre les actions du gouvernement pour développer la création d'entreprise et l'apparition de nouvelles valeurs dans la population. Dans l'étude menée par Nyock Ilouga et al. (2013), le soutien social (eg. la famille) exerce une influence positive sur l'entrepreneuriat. Ainsi, au-delà des facteurs de motivation internes, d'autres facteurs d'ordre externe (culturels, économiques, sociaux) vont motiver la création d'entreprise.

\section{La génération $Y$ : caractéristiques et entrepreneuriat}

Il n’y a pas un clair consensus concernant la définition de la génération Y (Minifie, Otto, 2011 ; Pichault, Pleyers, 2010, Tremblay et al., 2010). Différents noms ont été utilisés pour identifier les jeunes d'aujourd'hui tels que «Génération Internet», «Echo Boomers», «E-génération», «Millenials», «Gen Y», «Nexters», etc. (Brillet et al., 2012). Plusieurs dates sont également proposées pour délimiter cette génération : 1978/1990 (Tremblay et al., 2010), 1978/1996 (Bayad et al., 2013), 1980/2000 (Eastman et al., 2014 ; Farrell, Hurt, 2014, Gibson, Sodeman, 2014). Certains articles questionnent même la réelle différence qui justifierait l'identification des jeunes d'aujourd'hui comme appartenant à une nouvelle génération (Pichault, Pleyers, 2010 ; Bayad et al., 2013). Toutefois, au-delà du manque de précision par rapport au nom ou à la date de naissance, la plupart des articles publiés dans les médias s'accordent sur le fait que la jeunesse actuelle est relativement différente de celles d'autres époques. Cette différence a été stéréotypée et concerne des aspects tels que la relation à l'autorité (indépendance), la relation au temps (impatience) et l'importance accordée aux loisirs et à la vie privée (au détriment du travail) (Bayad et al., 2013). Un autre trait distinctif de 
cette génération concerne les aspects paradoxales associés aux jeunes d'aujourd'hui : individualistes mais avec un intérêt communautaire, orientés vers le succès mais sans délaisser la vie privée et les loisirs, n'acceptant pas la hiérarchie mais cherchant constamment le feedback. Aussi, d'après la littérature, la génération $\mathrm{Y}$ est technologique et multitâches, et cherche attention et feedback continuellement (Farrel, Hurt, 2014 ; Gibson, Sodeman, 2014 ; Holt et al., 2012). L'étude faite par Claire (2012) montre que la réussite économique est importante, mais que le succès est une mesure complexe qui va au-delà du profit. Cette génération a pour caractéristiques principales : le fait d'aimer les défis, de chercher la croissance personnelle, de développer un impact positif et d'aider les autres, de réussir et d'avoir une stabilité financière (Holt et al., 2012). L'étude menée par Minifie et Otto (2011) va dans le même sens, en rajoutant le désir de flexibilité, de travailler de façon intelligente («work smart rather than hard»), la recherche d'un équilibre travail-famille, et le fait que «faire » est plus important que « savoir ». A partir de ces résultats, les auteurs concluent avec l'importance d'une pédagogie centrée sur l'action, du type «learning by doing » ou « experiential learning », quand il s'agit des jeunes d'aujourd'hui.

Les études sur l'entrepreneuriat des jeunes millénaires sont émergentes (Bayad et al., 2013), contrairement aux études en marketing ou en ressources humaines. En effet, la littérature s'est d'abord intéressée aux caractéristiques des jeunes en tant que consommateurs (Eastman et al., 2014), ou en tant qu'employés (Farret, Hurt, 2014). Même si les enquêtes montrent un changement d'orientation professionnel chez les jeunes millénaires (lesquels ont moins tendance à postuler à des emplois salariés et lancent plus souvent leurs propres entreprises ${ }^{3}$ ), la littérature en entrepreneuriat ne compte que peu de travaux sur la création d'entreprise des jeunes Y (Tremblay et al., 2010). Concernant ce changement d'orientation professionnelle des jeunes, les résultats d'une enquête menée par Eddy $\mathrm{Ng}$ et al. (2010) montrent que la moitié des jeunes répondants n'étaient pas intéressés par un poste en entreprise, ceci étant une conséquence du travail intensif des babyboomers, associés également aux restructurations d'entreprises et aux licenciements qu'ils ont subi.

\footnotetext{
${ }^{3}$ http://www.huffingtonpost.com/marty-zwilling/tips-for-gen-y-on-how-to b 3958917.html (consulté le 22/06/2015)
} 
Par ailleurs, les jeunes millénaires semblent ambitieux et impatients, cherchent des opportunités pour avancer et visent à réussir un équilibre entre la vie privée et le travail ( $\mathrm{Ng}$ et al., 2010). Ainsi, d'après American Express OPEN Ages Survey ${ }^{4}$, la raison principale par laquelle les jeunes de la génération Y deviennent entrepreneurs est la passion, et leur satisfaction est principalement liée à leurs relations d'amitié et de famille.

Munis de motivations différentes, et dans un contexte différent, les entreprises créées par les millénaires s'éloignent de l'ancien idéal d'entreprise (Claire, 2012). Elles sont plutôt flexibles, petites, connectés, avec peu de capital de lancement et peu d'employés, et permettent à l'entrepreneur de travailler où et quand il le désire ${ }^{5}$. Néanmoins, les études qui comparent l'entrepreneuriat des jeunes de la génération Y à celui des individus de la génération $\mathrm{X}$, ou même les baby-boomers, créant des entreprises aujourd'hui, ne trouvent pas beaucoup de différences (Bayad et al., 2013). La recherche d'une vie équilibrée, avec du temps pour le travail, la famille et les loisirs, est devenue dorénavant une valeur importante pour toutes les générations. Comme le disent Pichault et Pleyers (2010), les trois générations partagent des motivations entrepreneuriales relativement similaires.

\section{La méthodologie}

Dans les paragraphes qui suivent, nous allons présenter successivement l'approche méthodologique, la collection des données et l'analyse effectuée.

\subsection{L'approche méthodologique}

Dans cette recherche, nous avons mis en place une approche qualitative exploratoire, basée sur la méthode de l'étude de cas. La littérature nous indique que ce choix est cohérent lorsque l'objectif est : (1) de révéler un phénomène existant encore faiblement étudié par la communauté scientifique

\footnotetext{
${ }^{4}$ http://www.businesswire.com/news/home/20130805006053/en/Generational-Divide-Passion-Fuels-PerseveranceGen-Entrepreneurs\#.UtTtm7S0jk8 (consulté le 22/06/2015).

${ }^{5}$ http://www.huffingtonpost.com/marty-zwilling/tips-for-gen-y-on-how-to b 3958917.html (consulté le 22/06/2015)
} 
(Yin, 1994) ; (2) de décrire un nouveau phénomène qui mérite l'attention (Siggelkow, 2007) ; ou (3) de construire de nouvelles théories (Eisenhardt, Graebner, 2007). Il permet également d'ancrer les phénomènes étudiés dans la réalité et d'identifier des liens entre un phénomène particulier et ses causes potentielles (Dumez, 2013). Cette méthode est caractérisée par une validité externe limitée qui réduit sa capacité de généralisation statistique et une faible fiabilité des données produites (Thietart, 2007). Néanmoins, même si l'étude est réalisée sur un seul cas, la méthode est considérée comme pertinente (Mauss, 1966). Pour Eisenhardt et Graebner (2007), l'utilisation d'études de cas fournit une lecture qualitative d'un phénomène et permet une théorisation abductive ancrée dans des données empiriques.

Nous pensons que cette approche est pertinente dans le cadre de notre recherche car, à ce jour, la problématique des étudiants entrepreneurs a été peu explorée dans la littérature académique. Notre choix a été justifié par notre intention de comprendre les valeurs et les motivations des étudiantsentrepreneurs en école de commerce.

Les cas étudiés sont des étudiants-entrepreneurs en master entrepreneuriat dans une école de commerce en région parisienne. L'école propose une formation ainsi qu'un accompagnement des projets en création et les étudiants créent régulièrement des entreprises, tout au long du déroulement de leurs études. L'école est connue pour sa vocation entrepreneuriale et compte sur des places disponibles pour incuber les projets des étudiants dans trois incubateurs différents à Paris. Les étudiants peuvent aussi être financés par un fond d'amorçage géré par les anciens de l'école.

\subsection{La collection de données}

Afin d'explorer les valeurs et motivations portées par les jeunes étudiants-entrepreneurs, nous avons décidé d'interviewer tous les étudiants en dernière année du master management entrepreneurial qui avaient déjà créé leurs entreprises. L'objectif des chercheurs était alors d'obtenir, non pas un nombre important d'entretiens, mais de faire une étude exhaustive d'une population particulière. Au final, sept étudiants-entrepreneurs remplissaient ces conditions. Nous les avons identifié comme appartenant à la génération Y. Pour le faire, nous avons suivi les classifications d'Eastman et al. 
(2014) et de Farrell et Hurt (2014), qui proposent la période 1980-2000 pour délimiter à la génération Y. Ainsi, les jeunes entrepreneurs de notre échantillon se placent au milieu de cette période (nés entre les années 1989 et 1992). Notre échantillon est composé comme il suit :

Tableau 1: Les participants

\begin{tabular}{|l|l|l|}
\hline Noms & Age & Secteurs \\
\hline I1 & 22 & Technologique \\
\hline I2 & 24 & Technologique \\
\hline I3 & 22 & Technologique \\
\hline I4 & 23 & Art/Mode \\
\hline I5 & 25 & Evénementiel \\
\hline I6 & 22 & Technologique \\
\hline I7 & 22 & Relations publiques \\
\hline
\end{tabular}

L'échantillon est mixte, avec une prépondérance de jeunes entrepreneurs $\mathrm{du}$ sexe masculin (uniquement I2 et I4 sont des femmes). La collecte des données a été effectuée sur la base d'entretiens approfondis (Miles, Huberman, 1994). Les entretiens approfondis sont particulièrement adaptés lorsque le chercheur vise à mettre en avant de nouvelles connaissances (Merton, Fiske, Kendall, 1990 ; Ettl, Welter, 2010). Les entretiens sont ainsi un outil à privilégier pour obtenir des informations sur des sujets complexes et peu explorés (Merton, Fiske, Kendall, 1990 ; Evrard, Roux, Pras, 1992 ; Liberman-Yaconi, Hooper, Hutchings, 2010).

Les entretiens approfondis ont été menés en suivant la méthode du récit de vie. Pour cela, nous avons demandé aux interviewés de nous faire part de leur expérience entrepreneuriale. Nous avons porté une attention particulière aux facteurs de motivation dans leur décision de créer une entreprise. Nous avons aussi demandé aux interviewés de définir les valeurs principales qui, d'après eux, représentent mieux leur génération. Les entretiens ont duré entre 45 minutes et une heure. Ils ont été effectués en face à face durant l'année 2013. Nous avons aussi collecté des informations variées concernant les entreprises créées, et sur le profil des étudiants-entrepreneurs, afin de trianguler les données récupérées.

\subsection{L'analyse des données}


Les entretiens ont été enregistrés, intégralement retranscrits, et analysés suivant une approche thématique manuelle (Evrard et al., 1997). L'étude a suivi une démarche abductive comme proposée par la théorie enracinée (Strauss et Corbin, 2004). Ainsi, l'analyse commence dès le premier entretien et les concepts sortent du terrain. Le codage évolue de manière itérative, au fur et à mesure des entretiens, avec des allers-retours entre le terrain et la théorie. De cette manière, dans un premier temps, nous avons analysé les discours obtenus pour en faire sortir les concepts pertinents et, dans un deuxième temps, nous avons organisé ces derniers, ainsi que leurs relations entre eux.

L'analyse de contenu, qui consiste à «lire un corpus, fragment par fragment, pour en définir le contenu en le codant selon des catégories qui peuvent être construites et améliorées au cours de la lecture »(Fallery, Rodhain, 2007) a permis d'identifier les verbatims représentant les différents facteurs motivationnels, aussi bien internes qu'externes, et la perception des valeurs exprimées par les interviewés. Le tableau 2 résume le codage des verbatims.

Tableau 2 : Codage des verbatims

\begin{tabular}{|c|c|c|c|}
\hline \multirow{15}{*}{$\begin{array}{l}\text { Dimensions } \\
\text { Motivation }\end{array}$} & \multirow{6}{*}{ Facteurs internes } & \multirow{2}{*}{ (In)satisfaction } & Frustration salariat (MIs-FS) \\
\hline & & & Passion (MIs-P) \\
\hline & & \multirow{2}{*}{ Opportunité } & Economique (MO-E) \\
\hline & & & Economique modérée (MO-EM) \\
\hline & & Nécessité & Chômage (MN-C) \\
\hline & & Indépendance & Autonomie (MId-A) \\
\hline & \multirow{9}{*}{ Facteurs externes } & \multirow{2}{*}{ Environnement Social } & Soutien (MES-S) \\
\hline & & & Role Model (MES-RM) \\
\hline & & \multirow{3}{*}{ Contexte Economique } & Taux de chômage (MCE-TC) \\
\hline & & & Instabilité (MCE-I) \\
\hline & & & Conditions de travail (MCE-CT) \\
\hline & & \multirow{4}{*}{$\begin{array}{c}\text { Environnement } \\
\text { Politico/Institutionnels }\end{array}$} & Aides financières (MEP-A) \\
\hline & & & Démarches faciles (MEP-DF) \\
\hline & & & Incubation (MEP-I) \\
\hline & & & Ecoles (MEP-E) \\
\hline \multirow{12}{*}{$\begin{array}{l}\text { Dimensions } \\
\text { Culturelles }\end{array}$} & \multirow{12}{*}{ Valeurs } & \multirow{6}{*}{ Travail } & Rejet salariat (VT-RS) \\
\hline & & & Travailler pour vivre (VT-TV) \\
\hline & & & Gagner pour vivre (VT-GV) \\
\hline & & & Confort (VT-C) \\
\hline & & & Épanouissement (VT-E) \\
\hline & & & Conciliation vie privée (VT-CVP) \\
\hline & & \multirow{4}{*}{ (In)stabilité } & Relations passagères (VS-RP) \\
\hline & & & Volatile (VS-V) \\
\hline & & & Mouvement (VS-M) \\
\hline & & & Multi-tâche (VS-Mt) \\
\hline & & \multirow{2}{*}{ Autorité } & Rejet hiérarchie (VA-RH) \\
\hline & & & Rejet stress (VA-S) \\
\hline
\end{tabular}


L'analyse du discours nous a permis d'identifier et de regrouper les facteurs motivationnels en deux catégories: internes (concernant les besoins personnelles de l'individu) et externes (en lien avec l'environnement de l'individu).

Les facteurs de motivation internes correspondent aux 4 dimensions de l'approche push/pull : nécessité, opportunité, insatisfaction et recherche d'indépendance. En suivant le texte de Gabarret et Vedel (2015), nous avons considéré les dimensions comme continus. Ainsi, dans la dimension satisfaction, nous avons codé tous les verbatims déclarant une insatisfaction au travail, mais aussi ceux déclarant une recherche de satisfaction, et parmi cette dernière la "passion » d'entreprendre. Par ailleurs, comme les étudiants n'avaient pas (ou peu) d'expérience antérieure d'emploi, le facteur d'insatisfaction a été élargi à l'insatisfaction avec le travail salarié (perception) et non avec une réelle insatisfaction par rapport à une expérience préalable. De la même manière, le facteur « indépendance » correspond aussi bien à un manque d'indépendance qu'à une recherche d'indépendance. L'opportunité est généralement considérée d'un point de vue économique comme une possibilité de maximisation de bénéfices (opportunité de gagner davantage). Nous l'avons considéré aussi d'un point de vue économique modéré (opportunité d'affaires qui permet de gagner de l'argent pour vivre et non de vivre pour gagner de l'argent). Finalement, la nécessité est considérée comme un manque de travail (chômage) ou comme un besoin de travail.

Au-delà des facteurs de motivation internes, nous avons également identifié et classé des concepts émergents correspondants à l'influence de l'environnement dans lequel la création d'entreprise a eu lieu. Trois groupements de facteurs ont été codés : sociales (soutien et role model), économiques (chômage, instabilité économique et conditions de travail) et politico-institutionnels (aides financières, facilité des démarches, incubation et écoles). Les concepts mobilisés par les interviewés font référence à des facteurs externes, tels qu'expliqué par Williams et Williams (2012). Les auteurs indiquent que la motivation entrepreneuriale peut être influencée par le contexte socio-spatial. Ainsi, 
nous avons créé des catégories contextuelles afin de pouvoir classer les facteurs de motivation externes repérées.

Une étude culturelle a été aussi développée, à travers l'identification des valeurs partagées par les interviewés dans leur discours. Le langage utilisé par un individu renferme les valeurs qu'il veut transmettre (Bourdieu, 1991). Ces valeurs le conduisent à l'action grâce au sens qu'ils portent (Weick, 1979), le langage servant de moyen pour coordonner les actions. La culture peut être considérée comme une collection de valeurs partagées, qui modèle le comportement et définie ce qui est désirable pour un groupe social (Schwartz, 1999).

Concernant les valeurs cachées dans le discours motivationnel, trois concepts principaux ont été codés : le travail, la stabilité et l'autorité. Pour la valeur donnée au «travail » nous avons regroupé des verbatims représentant les aspects suivants : (1) vouloir travailler pour vivre et non vivre pour travailler, (2) vouloir gagner de l'argent pour vivre bien et non pour faire fortune, (3) adapter le travail à une recherche de confort, (4) adapter le travail à une recherche d'épanouissement, et (5) chercher la conciliation vie privée-travail. Pour comprendre la valeur donnée à la« stabilité » nous avons classé les verbatims suivants : (1) le caractère passager des relations interpersonnelles, (2) la volatilité du comportement des jeunes, (3) le fait qu'ils cherchent à être en mouvement tout le temps sans vouloir se poser, et (4) l'aspect multi-tâche (faire beaucoup de choses différentes en même temps). Finalement, pour comprendre la valeur donnée à l'«autorité » nous avons regroupé les verbatims concernant les rejets de la hiérarchie et du stress provoqué par les relations d'autorité.

La motivation entrepreneuriale, considérée comme complexe (Kirkwood, Cambell-Hunt, 2007), est liée aussi bien à des facteurs internes qu'externes, dont, par exemple, les politiques gouvernementales qui permettent l'apparition de nouvelles valeurs (Liñan et al., 2013). Ainsi, nous nous sommes focalisés sur les verbatims qui correspondaient aux valeurs culturelles. Les aspects identifiés sont cohérentes avec les études mobilisées sur les valeurs de la génération Y, tels que celle de Bayad et al. (2013). En effet, d'après ces auteurs, la génération Y a été stéréotypée selon plusieurs 
axes : la relation à l'autorité (indépendance), la relation au temps (impatience) et l'importance accordée aux loisirs et à la vie privée (au détriment du travail) (Bayad et al., 2013).

\section{Résultats et discussion}

Dans les sections suivantes, nous allons présenter les résultats de notre étude qualitative en deux volets : les motivations entrepreneuriales et les valeurs perçues.

\subsection{Les dimensions de la motivation entrepreneuriale chez les jeunes $Y$}

La classification des facteurs de motivation est présentée dans le tableau 3

Tableau 3: Les facteurs motivationnels

\begin{tabular}{|c|c|c|c|c|c|c|c|}
\hline & \multicolumn{4}{|c|}{ Facteurs de motivation internes } & \multicolumn{3}{c|}{ Facteurs de motivation externes } \\
\hline & $\begin{array}{c}\text { (In)sati- } \\
\text { sfaction }\end{array}$ & $\begin{array}{c}\text { Indépen- } \\
\text { dance }\end{array}$ & $\begin{array}{c}\text { Opportu- } \\
\text { nité }\end{array}$ & Nécessité & Sociaux & $\begin{array}{c}\text { Economi- } \\
\text { ques }\end{array}$ & $\begin{array}{c}\text { Politico- } \\
\text { Institu- } \\
\text { tionnels }\end{array}$ \\
\hline $\mathrm{I} 1$ & & $\mathrm{X}$ & $\mathrm{X}$ & & $\mathrm{X}$ & & $\mathrm{X}$ \\
\hline $\mathrm{I} 2$ & $\mathrm{X}$ & & $\mathrm{X}$ & & $\mathrm{X}$ & $\mathrm{X}$ & \\
\hline $\mathrm{I} 3$ & $\mathrm{X}$ & $\mathrm{X}$ & $\mathrm{X}$ & & $\mathrm{X}$ & $\mathrm{X}$ & $\mathrm{X}$ \\
\hline $\mathrm{I} 4$ & $\mathrm{X}$ & $\mathrm{X}$ & $\mathrm{X}$ & & $\mathrm{X}$ & $\mathrm{X}$ & $\mathrm{X}$ \\
\hline $\mathrm{I} 5$ & & $\mathrm{X}$ & & & $\mathrm{X}$ & & \\
\hline $\mathrm{I} 6$ & $\mathrm{X}$ & $\mathrm{X}$ & & & $\mathrm{X}$ & & \\
\hline $\mathrm{I} 7$ & $\mathrm{X}$ & $\mathrm{X}$ & & & $\mathrm{X}$ & $\mathrm{X}$ & $\mathrm{X}$ \\
\hline
\end{tabular}

Une première approche nous montre que les jeunes étudiants-entrepreneurs sont motivés par une combinaison de dimensions push et pull (Verheul et al., 2010). De plus, la plupart des jeunes entrepreneurs interviewés ont exprimé des concepts liés à des dimensions motivationnelles internes et externes. Parmi les facteurs internes, les étudiants-créateurs manifestent une composition d'aspects d'insatisfaction, de recherche d'indépendance et d'opportunité d'affaires. Nous n'avons pas repérée des verbatims liés à la création d'entreprise par nécessité dans cet échantillon. Néanmoins, l'absence de facteurs de nécessité dans notre terrain doit être considérée avec précaution. En effet, ils ont créé leurs entreprises pendant leurs années de scolarité et n'ont pas eu l'occasion de se confronter au marché du travail. 
Concernant la mesure de l'(in)satisfaction au travail, comme expliqué dans la partie méthodologie, cette dimension couvre aussi bien l'insatisfaction que la recherche de satisfaction. Nous avons dû élargir le concept d'insatisfaction au-delà de la simple expérience professionnelle, car, en étant étudiants, cette dernière est limitée à quelques stages effectués tout au long de leur période de scolarité. Il apparaît ainsi que les individus interrogés déclarent ne pas avoir une impression positive par rapport à l'emploi salarié en général. Cela est dû, d'une part, à la représentation négative qu'ils ont de l'environnement macroéconomique du pays (la France possède un taux relativement important de chômage et très peu de croissance). D'autre part, ils partagent la perception que même s'ils trouvent un travail salarié, celui-ci risque de ne pas être satisfaisant: "Je n'ai pas vraiment d'expérience dans les grandes organisations, mais toutes ces grosses structures laissent à penser qu'on a beau s'investir il n'y a pas de retour là-dessus » (I4) ; «Les entreprises en plus quand tu es jeune te font travailler beaucoup et ne paient que très peu, elles t'exploitent pas mal»(I3). Concernant la recherche de satisfaction, les entrepreneurs ont expliqué leur désir de suivre une passion, ou d'avoir un travail qui leur procure du plaisir, de la satisfaction : «J'ai eu la volonté de créer un projet autour d'une passion et aussi la volonté de ne pas être salarié plus tard»(I7), «Dans l'ordre, ce serait plus la passion (...), dans un second temps ça serait la liberté d'action et dans un troisième temps ce serait l'argent »(I3). Nos résultats coïncident, d'une part, avec les études développées par Eddy Ng et al. (2010), qui montrent le manque d'intérêt des jeunes pour une carrière traditionnelle, et, d'autre part, avec l'enquête d'American Express OPEN Ages, dans laquelle les jeunes entrepreneurs manifestent créer des entreprises principalement par passion.

Une deuxième dimension motivationnelle présente dans les entretiens est celle d'un désir d'indépendance (ie. être libre, être autonome, gérer sa propre vie...) : «Depuis le lycée, (...) j’ai juste envie d'être indépendante »(I4), «Je n'avais pas envie d'être salarié, j'ai commencé pour le plaisir de faire et j'ai vu que je pouvais faire de l'argent (...) c'est la liberté de faire ce que je veux, d'avoir mes propres objectifs»(I5). Le désir d'indépendance, au-delà d'être une motivation 
principale des entrepreneurs de tout âge, il est aussi une caractéristique importante de la nouvelle génération (Bayad et al., 2013).

Avoir trouvé une opportunité d'affaires est aussi mentionné par les étudiants-entrepreneurs. En général, dans la littérature, la dimension «opportunité » est en relation avec une amélioration du niveau de revenu (Kelley et al., 2011). Néanmoins, les jeunes Y interrogés ne pensent pas que cette opportunité leurs permettra de créer une valeur économique importante : "l'entrepreneuriat n'est pas le meilleur chemin pour devenir millionnaire » (I4) ; «Mon troisième objectif est de gagner de l'argent mais c'est sur le long terme, d'ici 10 ou 15 ans (I3). Ainsi, il semble que la recherche d'enrichissement économique, au travers de la découverte d'une opportunité d'affaires, est plus modérée. Ce constat se rapproche des résultats de l'étude menée par Claire (2012), dans laquelle la réussite économique perd de l'importance face à d'autres mesures de réussite telles que l'éthique, ou les relations humaines. $\mathrm{Ng}$ et al. (2010) montrent également que les jeunes $\mathrm{Y}$ cherchent des opportunités pour avancer mais en respectant un équilibre vie privée-travail.

Les aspects motivationnels externes, d'après le discours des étudiants-créateurs, ont été regroupés en trois catégories (sociales, économiques et politico-institutionnelles). Concernant les facteurs sociaux, tous les individus ont manifesté avoir été motivés par la présence de role-models (famille, amis, ...). En effet, les entrepreneurs de l'environnement proche jouent le rôle d'exemple : « J'ai eu un exemple de réussite entrepreneuriale dans mon entourage et c'est vrai que ça me donne aussi l'envie de créer et d'avoir la satisfaction de pouvoir me dire que j'ai réussi et que je peux devenir un exemple pour une future génération » (I1) ; «J'avais mon grand-père qui était entrepreneur, (...) il m'a toujours inculqué ses valeurs entrepreneuriales »(I2). Dans notre texte, l'exemplification sociale peut avoir aussi bien un rôle direct qu'inverse. Ainsi, et comme nous l'avons montré par rapport à la dimension d'insatisfaction, la perception négative de l'emploi salarié par rapport à la carrière professionnelle des parents, peut amener les jeunes étudiants à considérer l'entrepreneuriat comme une meilleure option de carrière. De cette manière, le role model «salarié d'entreprise » 
agira de manière inverse, ayant un impact positif sur la création d'entreprise. Si les jeunes Y considèrent que leurs parents ont fait beaucoup d'effort, ils vont créer leurs entreprises en rejetant le modèle de leurs parents : «Moi mes deux parents sont salariés, je sais très bien ce qu'est la situation contrairement aux personnes ayant des parents entrepreneurs. Je vois très bien mon père encore trimer à son boulot à 60 ans alors qu'il veut être à la retraite alors que je vois autour de moi des gens qui ont des parents entrepreneurs ils ont, entre guillemets, la belle vie »(I6). De plus, si leurs parents sont des entrepreneurs réussis, cela a une influence directe sur la motivation à la création. Au-delà de la présence de modèles à suivre, le fait de compter sur le soutien de la famille est une notion récurrente dans nos entretiens. Cela avait été déjà repéré dans l'étude de Nyock Ilouga et al. (2013) qui montre que le soutien social exerce une influence positive sur l'entrepreneuriat.

Parmi les facteurs économiques, un des plus cités est le problème du chômage des jeunes en France. Ces facteurs influencent la décision de se lancer de façon autonome : «La situation actuelle fait que les offres d'emploi sont limitées, (...) il y a plus d'opportunités si on créé son entreprise »(I2), «Moi je n'ai pas envie d'être dépendante du marché du travail, (...) ça revient encore à dire qu'on veut être acteur de notre vie, qu'on a envie de prendre les choses en main »(I4). Le problème du chômage des jeunes est considéré comme un facteur d'environnement et d'insatisfaction, mais il ne déclenche pas la création d'entreprise par nécessité. En effet, ces étudiants entrepreneurs ne voient pas la création d'entreprise comme une obligation pour obtenir les moyens à leur survie, mais comme un défi bien adapté à une réalité d'emploi. De cette manière les caractéristiques de l'environnement économique vont agir en faveur de la voie entrepreneuriale, tout comme les aspirations personnelles (Nyock Ilouga et al., 2013).

Dans la même optique, au niveau des facteurs politico-institutionnels, les mesures développées par le gouvernement pour aider à la création et pour faciliter les démarches (subventions, prêts d'honneur et accompagnement) servent de mécanismes incitatifs. Certains des entrepreneurs interrogés expliquent que la création d'entreprise en France est grandement facilitée : «Les entrepreneurs sont 
de plus en plus aidés, que ce soit au niveau des financements ou des conseils, et le fait de voir ça, ça m'a poussé et ça m'a aidé à entreprendre »(I1); «On se rend compte qu'on a beau se plaindre de la France, on est quand même bien accompagné dans notre démarche d'entrepreneurs »(I4). L'enseignement de l'entrepreneuriat dans les écoles de commerce ou à l'université sont des catalyseurs importants pour la création d'entreprise des jeunes : " L'école et l'exemple d'entrepreneuriat dans ma famille m'ont donné l'envie de créer »(I1), « Je suis arrivé en fin de terminale, je ne savais pas exactement ce que je voulais faire et j'ai trouvé l'école, et c'est là où je me suis mis dans la décision de devenir entrepreneur»(I3). Ceci rejoint les travaux de Liñan et al. (2013) sur la relation entre la motivation entrepreneuriale et les actions du gouvernement pour développer la création d'entreprise.

De cette manière les influences environnementales, telles que les exemples d'entrepreneurs réussis dans leur cercle de proximité, ainsi que la perception d'une facilité de création au travers des dispositifs mises à disposition des jeunes créateurs, vont se combiner avec les motivations de recherche d'indépendance (pull) pour les inciter à la création. Par ailleurs, la perception de la réalité économique difficile du marché du travail (chômage des jeunes) et les idées développés concernant la carrière en entreprise (insatisfaction par rapport au travail salarié) vont constituer des facteurs de poussée (push) qui vont aussi être déterminants de leur décision entrepreneuriale.

\subsection{La perception de valeurs et l'analyse du contexte culturel}

Au-delà de l'analyse de la motivation, nous avons complété la compréhension de l'entrepreneuriat des jeunes en rajoutant une analyse permettant d'étudier les valeurs qui se cachent derrière le discours motivationnel des jeunes Y interviewés. Comprendre les valeurs d'un groupe est une tâche difficile, ces dernières étant ancrées dans l'inconscient ${ }^{6}$. Nous pouvons, néanmoins, les étudier au travers des verbatims faisant référence aux attitudes et croyances, car ce sont les valeurs qui vont guider les comportements et les choix effectués par les individus. De cette manière, entremêlés dans

\footnotetext{
${ }^{6}$ La culture est généralement représentée comme un oignon, dont les comportements et attitudes sont dans les couches superficielles pendant que les valeurs sont dans le cœur de l'oignon (Trompenaars, 1996).
} 
le discours motivationnel, nous avons pu percevoir trois valeurs principales liées au travail, à la stabilité et à l'autorité (tableau 4).

Tableau 4 : Valeurs de la génération Y

\begin{tabular}{|l|c|c|c|}
\hline & Valeur Travail & Valeur (In)stabilité & Valeur Autorité \\
\hline $\mathrm{I} 1$ & $\mathrm{X}$ & $\mathrm{X}$ & \\
\hline $\mathrm{I} 2$ & $\mathrm{X}$ & & \\
\hline $\mathrm{I} 3$ & $\mathrm{X}$ & $\mathrm{X}$ & $\mathrm{X}$ \\
\hline $\mathrm{I} 4$ & $\mathrm{X}$ & $\mathrm{X}$ & $\mathrm{X}$ \\
\hline $\mathrm{I} 5$ & $\mathrm{X}$ & $\mathrm{X}$ & \\
\hline $\mathrm{I} 6$ & $\mathrm{X}$ & $\mathrm{X}$ & \\
\hline $\mathrm{I} 7$ & $\mathrm{X}$ & $\mathrm{X}$ & \\
\hline
\end{tabular}

Les jeunes-entrepreneurs de la génération Y n'ont pas une relation passionnelle avec le «travail ». En effet, ils préfèrent travailler pour vivre et non vivre pour travailler (Minifie, Otto, 2011). Le travail doit servir à gagner l'argent nécessaire pour vivre confortablement. De plus, la recherche d'une conciliation entre la vie privée et la vie professionnelle ( $\mathrm{Ng}$ et al., 2010 ; Minifie, Otto, 2011 ; Bayad et al., 2013) aide à réduire l'impact du travail sur la satisfaction de l'individu.

- « Le travail salarié existe mais c'est très dur (...) l'objectif ce n'est pas forcément de gagner beaucoup d'argent, c'est avoir de l'argent, pouvoir en profiter, ne manquer de rien »(I1).

- «L'argent?, oui, mais principalement pour vivre, en faisant ce qu'on veut faire »(I3).

- "Je rêve d'avoir une petite structure dans un pays où il fait beau, où il fait chaud (...) mon but ce n'est pas d'avoir une villa et une grosse voiture, c'est d'avoir de l'argent de côté pour pouvoir faire ce que je veux quand je le veux » (I6).

Un autre aspect qui ressort du discours des interviewés est celui de la «(in)stabilité ». Aujourd'hui, le mode de vie des jeunes les mène à développer des relations passagères. Ceci rejoint certaines caractéristiques décrites par plusieurs auteurs telles que leur volatilité, leur envie de rester en mouvement et leur caractère multi-tâche (Holt et al., 2012 ; Farrel, Hurt, 2014 ; Gibson, Sodeman, 2014).

- «Les relations interpersonnelles se créent facilement, mais elles ne sont pas réelles »(I5).

- «Les relations humaines sont diversifiées et plus superficielles »(I4).

- «On a plus d'opportunités, on bouge beaucoup plus, mais on n'a pas de vraies relations humaines $\gg$ (I6).

Un troisième aspect répertorié concerne le lien à l'«autorité ». Les jeunes Y fuient les relations d'autorité et les environnements stressants. Cela est aussi traduit par la frustration partagée par rapport à l'emploi salarié. Le rejet de l'autorité est ainsi lié au désir d'indépendance repéré dans notre 
étude des facteurs de motivation. En effet, comme le montrent Pichault et Pleyers (2010), les Y vont mener leurs projets de manière autonome et créative.

- «J'appartiens à une génération qui a du mal avec l'autorité »(I4).

- «Je n'ai pas envie d'avoir un patron, d'être récompensé par un patron (...) j'ai des problèmes avec l'autorité, je travaille mal sous la pression des autres » (I6).

\section{Conclusion}

Face à l'importante quantité d'articles dans les médias à propos de la génération $\mathrm{Y}$, et à l'augmentation de l'entrepreneuriat des jeunes, nous avons voulu explorer les motivations entrepreneuriales et les valeurs des jeunes étudiants-entrepreneurs. En effet, la création d'entreprise par des jeunes étudiants est un sujet d'actualité, de plus en plus d'institutions d'enseignement mettent en place des formations en entrepreneuriat. Ainsi, suite à une revue de littérature en entrepreneuriat sur la génération $\mathrm{Y}$, et à des entretiens semi-directifs exhaustifs des étudiants-entrepreneurs en dernière année de master entrepreneuriat d'une grande école de commerce à Paris, nous avons pu dresser un premier portrait de l'étudiant-entrepreneur. Les jeunes étudiants créateurs sont motivés par un mix de dimensions push et pull, mais aussi par un mix de motivations internes (propres à l'individu) et externes (en relation à l'environnement). Il apparaît que ces motivations sont cohérentes avec les valeurs perçues chez les jeunes de la génération Y.

Parmi nos résultats principaux, les étudiants-entrepreneurs manifestent une insatisfaction avec le travail salarié et une recherche de satisfaction matérialisée par le fait de pouvoir suivre leur passion. Le rejet du salariat est en relation avec les valeurs de (in)stabilité, d'autorité et de travail. De cette manière, l'instabilité des relations, le rejet des rapports hiérarchiques ou d'autorité et la place du travail dans la vie façonnent la dimension motivationnelle de satisfaction/insatisfaction des étudiantsentrepreneurs. En deuxième place, ils manifestent un désir d'indépendance. Cette recherche d'autonomie est en rapport avec la valeur d'autorité, et consiste à un rejet du stress provoqué par les rapports hiérarchiques. En troisième lieu, se place l'opportunité d'affaires. Néanmoins, comme nous avons montré dans nos résultats, cette dimension à caractère économique est de nature modérée. Cette dimension est cohérente avec la valeur travail. En effet, les jeunes manifestent vouloir 
travailler pour vivre et non le contraire. Ainsi, les relations personnelles et les loisirs concurrencent avec le travail pour arriver à un nouvel équilibre.

Au-delà des dimensions motivationnelles internes, nous avons pu repérer la présence de motivateurs externes dans leur décision de création. Principalement, nous trouvons des facteurs sociaux (soutien familial), ou la présence de modèles à suivre dans leur entourage ou dans les médias. En deuxième lieu, des facteurs macroéconomiques tels que le chômage des jeunes, ou la difficulté de trouver un poste accordé à ses goûts et besoins, fonctionnent comme des «démotivateurs » par rapport à la carrière traditionnelle, et favorisent la décision de création. Finalement, la perception d'avoir des aides financières, des démarches simplifiées (telles que le statut d'autoentrepreneur), l'accès à des services, des incubateurs, pépinières, et le soutien dans le cadre de l'école, sont des facilitateurs de la décision d'entreprendre.

Notre recherche confirme la complexité de la motivation entrepreneuriale (Kirkwood, Cambell-Hunt, 2007) et l'importance grandissante des motivations non économiques dans la création d'entreprise (Carsrud et Brannback, 2011). Elle montre aussi, d'une part, une relation entre la motivation entrepreneuriale et les valeurs des jeunes entrepreneurs (Liñan, 2013), et d'autre part, l'influence des facteurs environnementaux dans le développement de l'entrepreneuriat (Liñan, 2013 ; Nyock Ilouga et al., 2013). Cela rejoint l'idée de nombreux auteurs en entrepreneuriat à propos du besoin d'étudier la motivation entrepreneuriale en relation avec le contexte (Williams, Williams, 2012).

Dans une optique managériale, nos résultats suggèrent, premièrement, que les actions gouvernementales, telles que l'implantation de structures d'incubation, la mise en place du statut d'auto-entrepreneur et surtout l'utilisation d'un discours positif à l'égard de l'entrepreneuriat par les leaders politiques, peuvent avoir un impact sur le changement de valeurs et permettre de développer la motivation entrepreneuriale. Deuxièmement, la compréhension des facteurs de motivation et des valeurs affichées par des jeunes étudiants-entrepreneurs peut permettre d'avoir un regard critique par rapport aux programmes d'enseignement en entrepreneuriat (Tremblay et al., 2010). Pour que 
l'enseignement réussisse, il faut trouver une juste adéquation entre les informations transmisses et les attentes des étudiants. Les attentes, qui sont en relation avec leurs valeurs et motivations, devront se voir comblés de par l'information et l'accompagnement donné par l'école. Adapter l'enseignement et l'accompagnement aux nouvelles valeurs et aux nouvelles caractéristiques des jeunes entrepreneurs d'aujourd'hui, tout comme mettre à disposition des jeunes-créateurs des instruments d'aide à la création, semble primordial pour réussir le développement de l'entrepreneuriat des jeunes en France.

\section{Références}

ACS, Z, ARENIUS, P, HAY, M, MINNITI, M, 2005, Global Entrepreneurship Monitor, 2004 Executive Report, Babson College and London Business School.

ARENIUS, P, MINNITI, M, 2005, "Perceptual Variables and Nascent Entrepreneurship", Small Business Economics, Vol. 24, pp. 233-247.

BAYAD, M, AIT RAZOUK, A, KLEBER, A, 2013, "Peter Pan au pays de l'entrepreneuriat : apparition d'un mYthe ?", Actes du $8^{\text {ème }}$ Congrès de l'Académie de l'Entrepreneuriat et de l'Innovation, 22-25 Octobre, Fribourg.

BOURDIEU, P, 1991, Language and symbolic power, Cambridge, MA, Harvard University Press.

BRADLEY, D, ROBERTS, J, 2005, "Is Low Job Satisfaction a Cause of Self-Employment Entrance? A Survival Analysis Using Data from the British Household Panel Survey", working paper, East Carolina University, Greenville, North Carolina, 27 Mai.

BRILLET, F, COUTELLE, P, HULIN, A, 2012, "Quelles trajectoires professionnelles pour la génération Y ?", Gestion 2000, septembre - octobre, pp. 69-88.

BROCKHAUS, R, 1980, "The Effect of Job Dissatisfaction on the Decision to Start a Business", Journal of Small Business Management, Vol. 18, pp. 37-43.

CARSRUD, A, BRANNBACK, M, 2011, "Entrepreneurial Motivations: What Do We Still Need to Know?", Journal of Small Business Management, Vol. 49, n 1, pp. 9-26.

CLAIRE, L, 2012, “Re-Storing the Entrepreneurial Ideal: Lifestyle Entrepreneurs as Hero?”, Tamara Journal for Critical Organization Inquiry, Vol. 10, Issue 1, pp. 31-39.

DUMEZ, H, 2013, Méthodologie de la Recherche Qualitative, Vuibert, Paris.

EASTMAN, J, IYER, R, LIAO-TROTH, S, WILLIAMS, D, GRIFFIN, M, 2014, "The Role of Involvement on Millennials' Mobile Technology Be haviors: The Moder ating Impact of Status Consumption, Innovation, and Opinion Leadership", Journal of Marketing Theory and Practice, Vol. 22, n 4, pp. 455-470.

EISENHARDT, KM, GRABNER, ME, 2007, “Theory Building from Cases: Opportunities and Challenges", Academy of Management Journal, Vol. 50, pp.25-32.

ETTL, K, WELTER, F, 2010, "Gender, Context and Entrepreneurial Learning", International Journal of Gender and Entrepreneurship, Vol. 2, n², pp. 108-129.

EVRARD, Y, PRAS, B, ROUX, E, 1997, Market, études et recherches en marketing (2nd ed.), Nathan, Paris.

FALLERY, B, RODHAIN, F, 2007, "Quatre approches pour l'analyse de données textuelles : lexicale, linguistique, cognitive, thématique", XVIème Conférence Internationale de Management Stratégique, 6-9 juin, Montréal, Québec.

FARREL, L, HURT, A, 2014, "Training the Millennial Generation: Implications for Organizational Climate", Journal of Organizational Learning and Leadership, Spring, Vol. 12, n 1, pp. 47-60.

GABARRET, I, VEDEL, B, 2015, "Pour une nouvelle approche de la motivation entrepreneuriale", La Revue des Sciences de Gestion, Vol. 1, n 271, pp. 13-20. 
GIBSON, L, SODEMAN, W, 2014, "Millennials and Technology: Addressing the Communication Gap in Education and Practice, Organization Development Journal, Winter, pp. 63-75.

GILAD, B, LEVINE, P, 1986, “A Behavioral Model of Entrepreneurial Supply”, Journal of Small Business Management, October, pp. 45-53.

HESSELS, J, VAN GELDEREN, M, THURIK, R, 2008, "Entrepreneurial Aspirations, Motivations, and their Drivers", Small Business Economics, Vol. 31, pp. 323-339.

HLADY-RISPAL, M, 2002, La méthode des cas. Application à la recherche en gestion, De Boeck Supérieur, $256 \mathrm{p}$.

HOLT, S, MARQUES, J, WAY, D, 2012, "Bracing for the Millennial Workforce: Looking for Ways to Inspire Generation Y", Journal of Leadership, Accontability and Ethics, Vol. 9, n 6, pp. 81-93.

HUGHES, K, 2003, "Pushed or Pulled? Women's Entry into Self-Employment and Small Business Ownership", Gender, Work and Organization, Vol. 10, n 4, pp. 433-454.

KELlEY, D, BOSMA, N, AMOROS, J, 2011, Global Entrepreneurship Monitor 2010 Global Report, Babson College, Universidad del Desarrollo, London Business School.

KIRKWOOD, J, CAMBELL-HUNT, C, 2007, "Using Multiple Paradigm Research Methodologies to Gain New Insights into Entrepreneurial Motivations", Journal of Enterprising Culture, Vol. 15, n³ (September), pp. 219-241.

LIBERMAN-YACONI, L, HOOPER, T, HUTCHINGS, K, 2010, "Toward a Model of Understanding Strategic Decision-Making in Micro-Firms: Exploring the Australian Information Technology Sector", Journal of Small Business Management, Vol. 48, n 1, pp. 70-95.

LIÑAN，F，FERNANDEZ-SERRANO，J，ROMERO， I， 2013, "Necessity and Opportunity Entrepreneurship: The Mediating Effect of Culture", Revista de Economia Mundial, Vol. 33, pp. 21-47.

MAUSS, M, 1966, Sociologie et Anthropologie, PUF, Paris.

MERTON, R, FISKE, M, KENDALL, P, 1990, The focused interview: A manual of problems and procedures, New York: Free Press.

MILES, MB, HUBERMAN, AM, 1994, Qualitative Data Analysis: An Expanded Sourcebook, $2^{\text {nd }}$ ed., Sage Publications, Newbury Park, CA.

MINIFIE, J, OTTO, V, 2011, "Worker Fulfillment at the Service Industry Entry Level", Business Renaissance Quarterly, Vol. 6, $\mathrm{n}^{\circ}$ 1, pp. 29-38.

NG, ESW, SCHWEITZER, L, LYONS, S, 2010, "New Generation, Great Expectations: A Field Study of the Millennial Generation", Journal of Business Psychology, Vol. 25, pp. 281-292.

NYOCK ILOUGA, S, NYOCK, A, HIKKEROVA, L, 2013, "Influence des perceptions sociales sur l'intention entrepreneuriale des jeunes", Gestion 2000, Septembre-Octobre, pp. 115-130.

PICHAULT, F, PLEYERS, M, 2010, "Pour en finir avec la génération Y...Enquête sur une représentation managériale", XXIème Congrès AGRH, 17-19 Novembre, Rennes/St. Malo.

REYNOLDS, P, BYGRAVE, W, AUTIO, E, COX, L, HAY, M, 2002, Global Entrepreneurship Monitor, 2002 Executive Report, Babson College, Ewing Marion Kauffman Foundation, and London Business School.

SCHJOEDT, L, SHAVER, K, 2007, "Deciding on an Entrepreneurial Career: A Test of the Pull and Push Hypotheses Using the Panel Study of Entrepreneurial Dynamics Data", Entrepreneurship Theory and Practice, September, pp. 733-752.

SCHLAEGEL, C, HE, X, ENGLE, R, 2013, “The Direct and Indirect Influences of National Culture on Entrepreneurial Intentions: A Fourteen Nation Study", International Journal of Management, Vol. 30, $\mathrm{n}^{\circ}$ 2, part 2, pp. 597-609.

SCHWARTZ, S, 1999, "A Theory of Cultural Values and Some Implications for Work", Applied Psychology: An International Review, Vol. 48, $\mathrm{n}^{\circ}$ 1, pp. 23-47.

SIGGELKOW, N, 2007, "Persuasion with Case Studies", Academy of Management Journal, Vol. 50, $\mathrm{n}^{\circ} 1, \mathrm{pp} .20-24$.

STRAUSS, A, CORBIN, J, 2004, Les fondements de la recherche qualitative. Techniques et procédures de développement de la théorie enracinée, Academic Press Fribourg. 
THIETART, RA, 2007, Méthodes de Recherche en Management, 3rd ed., Dunod, Paris.

TREMBLAY, M, AUDET, J, GASSE, Y, 2010, "Aspirants entrepreneurs : le cas de la génération Y”, Document de travail 2010-001, Université Laval, Québec, Canada, disponible en ligne http://www5.fsa.ulaval.ca/sgc/documentsdetravail

TROMPENAARS, F, 1996, "Resolving International Conflict: Culture and Business Strategy", London Business School, Vol. 7, n 3, pp. 51-68.

VERHEUL, I, THURIK, R, HESSELS, J, VAN DER ZWAN, P, 2010, "Factors Influencing the Entrepreneurial Engagement of Opportunity and Necessity Entrepreneurs", SCALES, Scientific Analysis of Entrepreneurship and SMEs, march.

VERSTRAETE, T, SAPORTA, B, 2006, Création d'entreprise et entrepreneuriat, Les Editions de l'ADREG.

WEICK, K, 1979, The social psychology of organizing, Mcgraw Hill, New York, NY.

WILLIAMS, N, WILLIAMS, C, 2012, "Evaluating the socio-spatial contingency of entrepreneurial motivations: A case study of English deprived urban neighbourhoods", Entrepreneurship \& Regional Development, Vol. 24, $\mathrm{n}^{\circ}$ 7-8, September, pp. 661-684.

YIN, RK,1994, Case study research, Design and methods (3rd ed.), Thousand Oaks, CA. 